\title{
Optimal Hybrid Deconvolution Method for VLBI Images
}

\author{
Aidin Gharahdaghi ${ }^{1 *}$ and Omid Pakdelazar ${ }^{2}$ \\ ${ }^{1}$ Amirkabir University of Technology (AUT),Tehran, Iran; aidin.gharahdaghi@yahoo.com \\ ${ }^{2}$ Department of Electrical and Electronic Engineering, Iran University of Science and \\ Technology, Tehran, Iran; omidpakdelazar@gmail.com
}

\begin{abstract}
In this paper, we present a method for deconvolution of VLBI images based on both maximum entropy and compressive sensing concepts. The parameters of hybrid method are set optimally by utilization of particle swarm optimization (PSO) algorithm. The proposed method is also used to recover source image in a simulated VLBI. The capability of the proposed hybrid method in recovering the main information of target images of astronomical object is shown when the initial measurement data has limited quality. The main advantage of using such hybrid methods together with optimization is to take advantages from various methods in an integrated system.
\end{abstract}

Keywords: Very Long Baseline Interferometry, Maximum Entropy Method, Compressive Sensing, Particle Swarm Optimization.

\section{Introduction}

Radio astronomy has changed the history of human knowledge about outer space. Many discoveries became possible by utilizing radio telescopes through the $20^{\text {th }}$ and first decades of $21^{\text {th }}$ century [18]. However, the need for more resolution has resulted in the invention of VLBI (Very Long Baseline Interferometry) technique in recent decades. In VLBI an array of distant radio telescopes makes the effective aperture as big as needed. This array of telescopes makes some kind of interferometer by which it could be possible to make interferometric images of distant galaxies and other astronomical objects $[16,14]$. Recently, this technique has been utilized for observing and measuring event horizon of black holes [6,9]. The result of a VLBI observation is the sampled multi frequency transformation of image of the source represented in the $\mathrm{u}-\mathrm{v}-\mathrm{w}$ coordinate. Conventionally, by considering the $\mathrm{w}$ coordinate as perpendicular to the plane of interferometer, the resulted image is represented in orthogonal $\mathrm{u}-\mathrm{v}$ plane.
Because of limitations in sampling, both in time and spatial domain, some sort of after processing algorithms are necessary to make the images more utilizable. Inverse Fourier transform is not enough for gaining the best picture of distant observed radio source. Some deconvolution method is necessary to extract the best information from observed signal and its transformed versions. Basically, there will be dirty images to be input to a deconvolution algorithm whose output will be the goal image. Various algorithms are proposed to accomplish this task in recent years. The most basic one is CLEAN algorithm [5] in which iterative deconvolutions are performed on the dirty image to gain the final result. CLEAN algorithm needs some sort of user manipulation and is suitable mostly for lower quality and sparse data. Another proposed method for deconvolution of VLBI dirty images are based on maximization of entropy (Maximum Entropy Method, MEM) in which the image is estimated in a way that entropy measure could be maximized [1].The concept of entropy maximization is used in image processing in various application $[11,15]$.

\footnotetext{
* Corresponding author:

Aidin Gharahdaghi (aidin.gharahdaghi@yahoo.com)
} 
In contrast to CLEAN, MEM is suitable for better quality data with enough measurements to gain a better estimation for its distribution [1]. Recently proposed deconvolution algorithm based on compressed sensing (CS) method is developed to make use of concept of sparseness to overcome limits of sampling in VLBI imaging $[13,17]$. Some extensions to the CS is also presented in [19]. In this paper an optimal hybrid method is proposed for VLBI imaging. The proposed method takes the advantages of concepts from both CS and MEM methods. Optimality of the algorithm is achieved by using particle swarm optimization (PSO) method to set the optimum values for parameters of the hybrid algorithm. Firstly, as a part of introduction, the problem definition of this paper is described. In section 2, the basics of MEM, CS and PSO are presented. In section 3 , the proposed optimal hybrid method is introduced and section 4 discusses the results of its implementation on simulated images.

\subsection{Problem Definition}

In this paper the main problem is to design a deconvolution system which is used to extract an estimated image of a radio source from measurement data. The signals are assumed to be from measurements by an array of radio antennas on the Earth, and their signal correlations are calculated to take the advantages of interferometric method. The Earth rotation is also considered to have more diverse samples in $u v$ plane. The deconvolution system is a composition of CS and MEM concepts and the parameters for this system are to be determined by utilizing PSO. The performance of a design for the system is determined by recovering the source image appropriately.

\section{Components of Optimal Hybrid Method}

The proposed method of this paper is composed from three main components: 1. MEM, which is used to take advantages from information contents in the signals, 2. CS, which is the main part to overcome limits in sampling and sparseness of VLBI data, and 3. PSO, which optimizes the parameters of CS and MEM and their hybrid method.

\subsection{Maximum Entropy Method (MEM)}

One of the techniques for reconstructing a non-complete data, in our case an image, is to maximize an entropy measure by setting appropriate probability density and subject to some constraints. For VLBI images, the image $I(x, y)$ is reconstructed in a way that maximizes the entropy

$$
H=-\int d x d y I(x, y) \log \{(x, y)\},
$$

subject to the constraint that $I(x, y)$ should be close enough to the inverse Fourier transform of visibility function $V(u, v)$

$$
F\{(x, y)\}=V(u, v)+\eta .
$$

In basic cases, in literature the maximization task is performed based on method of Lagrange multipliers.

\subsection{Compressed Sensing (CS)}

In recent years the method of CS has found applications in different problems of signal processing like deconvolution and separation [7]. CS utilizes the concept of sparseness to reconstruct discrete Fourier transform and target signal. If most of coefficients in the Fourier transform of the signal are assumed to be zero, the signal is considered as sparse signal and CS could be applied to reconstruct unknown values. This statement is not only for Fourier transform but also applicable for other transformation which represent the signal in another basis such as wavelet transform. If discrete Fourier transform of image $I(x, y)$ is determined by matrix multiplication

$$
V(u, v)=D I(x, y),
$$

where the matrix $D$ is the product of two matrices: the measurement matrix $\psi$ and the projection matrix $\phi$,

$$
D=\Phi \psi .
$$

In CS it is also assumed that $\phi$ and $\psi$ areas incoherent as possible i.e.

$$
<\phi, \psi>\text { I }
$$

is minimum, where $\psi$ and $\phi$ are vectors from matrices $\phi$ and $\psi$. The remaining task is to find the sparsest signal by means of $\mathrm{L}_{0}$ or $\mathrm{L}_{1}$ norm minimization subject to incoherency assumption.

\subsection{Particle Swarm Optimization (PSO)}

Optimization methods based on evolution of a simulated system of some agents have found many applications 
in recent years. Different kinds of such methods are developed and each one has some sort of advantages related to other. Genetic Algorithms (GA) [10], Ant Colony Optimization (ACO) [8], and Particle Swarm Optimization (PSO) [12] are some of most utilized algorithms for optimization problems. Among them, PSO has the advantage of good compatibility with continuous variable problems. On the other hand, operations used in PSO are not time consuming and complex. In this section, the main procedure of PSO is presented for later use in optimization of parameters of hybrid deconvolution method. For more details and variations of PSO one can also see [2-4].

In PSO each possible solution of the optimization problem is represented as position vector of some particles in an $\mathrm{N}$ dimensional space, where $\mathrm{N}$ is the number of optimization variables. The optimization problem is to maximize or minimize a fitness function. The fitness function is the criterion based on the specific problem to be optimized and measures the fitness of a solution for that problem. Tendency to the best past position of each particle and the best position among all particles determines the movement of the particle in iterations of algorithm. Some kind of randomness is also applied to assure good search of solutions space by particles. This procedures result in gradual promotion of found solutions (as represented by particles best positions) regarding the fitness function. The main procedure of PSO is as follows:

1. Set predefined number of $\mathrm{N}$ dimensional vectors $x^{k}$ randomly to represent initial particles positions. Also set $\mathrm{N}$ dimensional initial velocity vector $v^{k}$ for those particles randomly.

2. Determine fitness of each particle regarding to predefined fitness function of problem.

3. Perform following operations iteratively:

(i) Name the best position among all particles as gbest, and name the best experienced position for each particle as pbest.

(ii) Update velocity of each particle using (1):

$$
\begin{aligned}
v^{k}(t+1)= & w v^{k}(t)+c_{1} r_{1}\left(\text { gbest }-x^{k}\right) \\
& +c_{\mathbf{2}} r_{\mathbf{2}}\left(\text { pbest }-x^{k}\right)
\end{aligned}
$$

where $w$ is inertia factor, $c_{1}$ and $c_{2}$ are constants and $r_{1}$ and $r_{2}$ are random numbers. (iii) Update particle positions by adding their updated velocity vector to them,

$$
x^{k}(t+1)=x^{k}(t)+v^{k}(t+1)
$$

(iv) Evaluate fitness for new particle positions.

(v) Check stopping criterion (e.g. some predefined maximum number of iterations) and decide to stop or continue the loop.

One of the tasks in utilization of PSO for a problem is to set appropriate parameters of the algorithm to have a good convergence and final fitness. One of the simplest methods to do so, which is also used in this research, is to tune the parameters by some trial and error. The maximum number of iterations for example is set in a way that after some number of iterations before that the result would not change.

In this paper PSO is utilized to find optimum values for parameters of the hybrid deconvolution method which is described in section 3 .

\section{Optimal Hybrid Method}

Two methods for deconvolution of VLBI images are introduced in sections 2-1 and 2-2, one based on entropy maximization and the other based on compressive sensing. These two approaches are applied well if some constraints about signal are satisfied. MEM is useful when data quality is reach enough and probability density could be estimated properly. On the other hand, CS is based on sparseness and incoherency assumptions. In this section, a hybrid method is proposed to take advantages from both MEM and CS. In the proposed method, a combination of objectives from both methods is set as the fitness function to be optimized. The optimization is accomplished by PSO. Related weights of objective terms are also determined by means of PSO. The main advantage of proposed hybrid method is that it could be applied to VLBI images without assumptions of the two discussed methods. The data could be sparse or non-sparse.

The fitness function for hybrid method is defined as

$$
f=-w_{1} H+w_{2} L_{1}+w_{3} \mu^{2}+w_{4}<\eta>^{2} .
$$

which should be minimized subject to closeness of visibility function to Fourier transform of dirty image, and when $w_{i}$ 's are positive valued. Generally, $w_{i}$ 's could 
be considered as functions of signal quality or other parameters E.g. proportional to the number of data points. To find the optimum values for parameters, PSO is used by considering them as particles positions. In the next section the proposed method is applied to simulated examples to show its advantage in VLBI images deconvolution.

\section{Simulation Results}

In this section we use optimal hybrid method to gain information about a source from very limited data of simulated VLBI imaging. We assume the radio source is an $\mathrm{X}$ shape object. We also assume 4 antennas scan the uv plane as shown in Figure 1. With this data and using earth rotation, visibility function and its direct inverse Fourier transform is obtained as shown in Figures 2 and 3 respectively.

Using PSO we find the image of source as shown in Figure 4, setting $w_{1}=0.88, w_{2}=0.07, w_{3}=0.48$ and $w_{4}=0.77$. The main characteristic of $X$ shape source is recovered by this method. Better quality of result will be obtained by optimizing antenna network topology.

In this research, simulated image is used to show the usefulness of method, and for this reason the source was to be taken as known. But the same procedure could be done for real VLBI images. The source used here is a

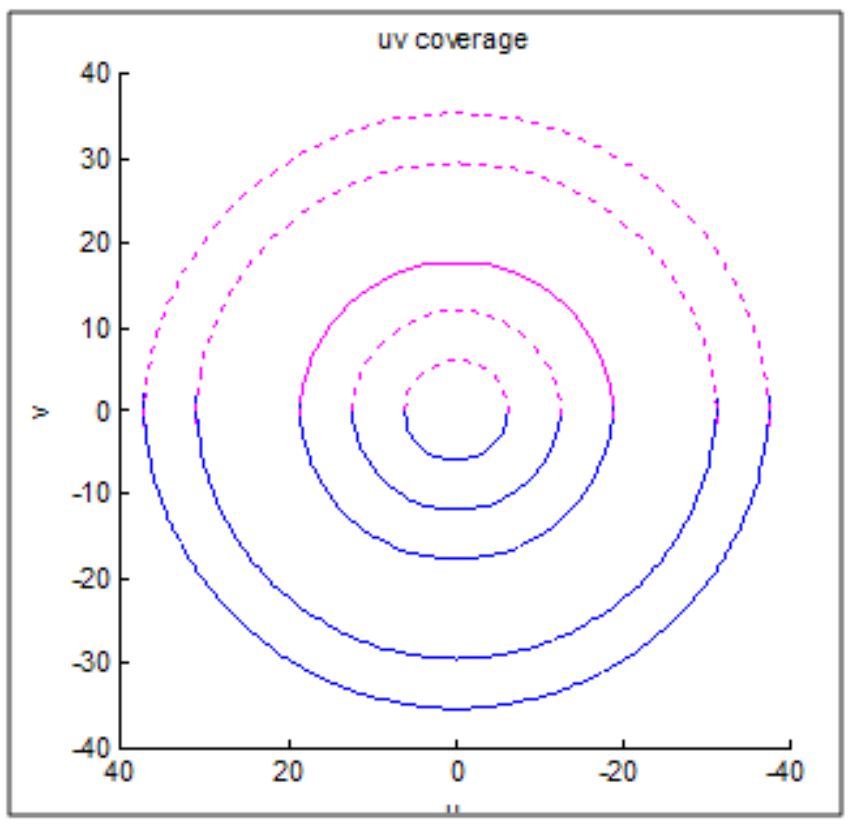

Figure 1. UV Scan profile of simulated VLBI.

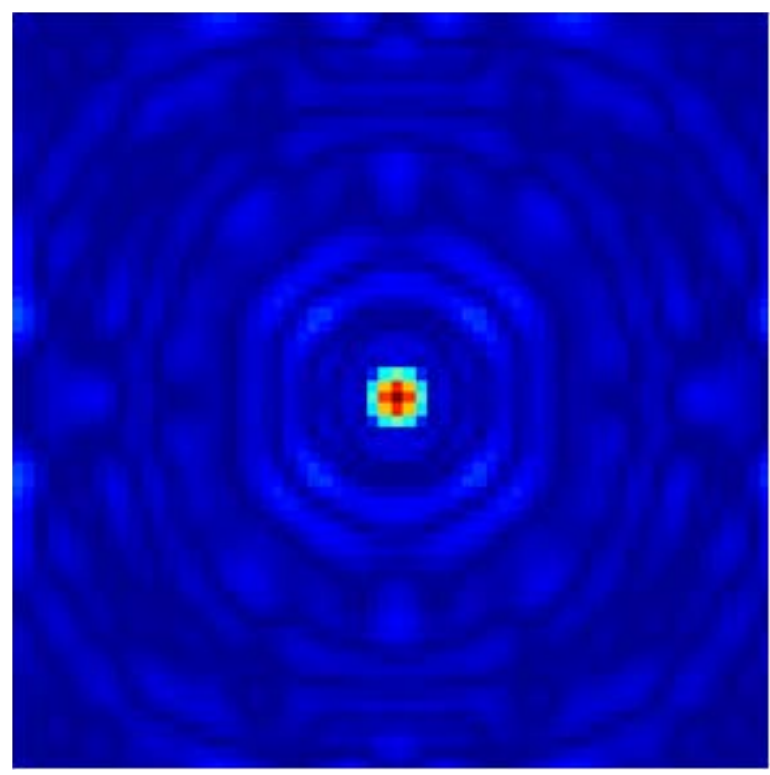

Figure 2. Visibility function obtained in uv plane.

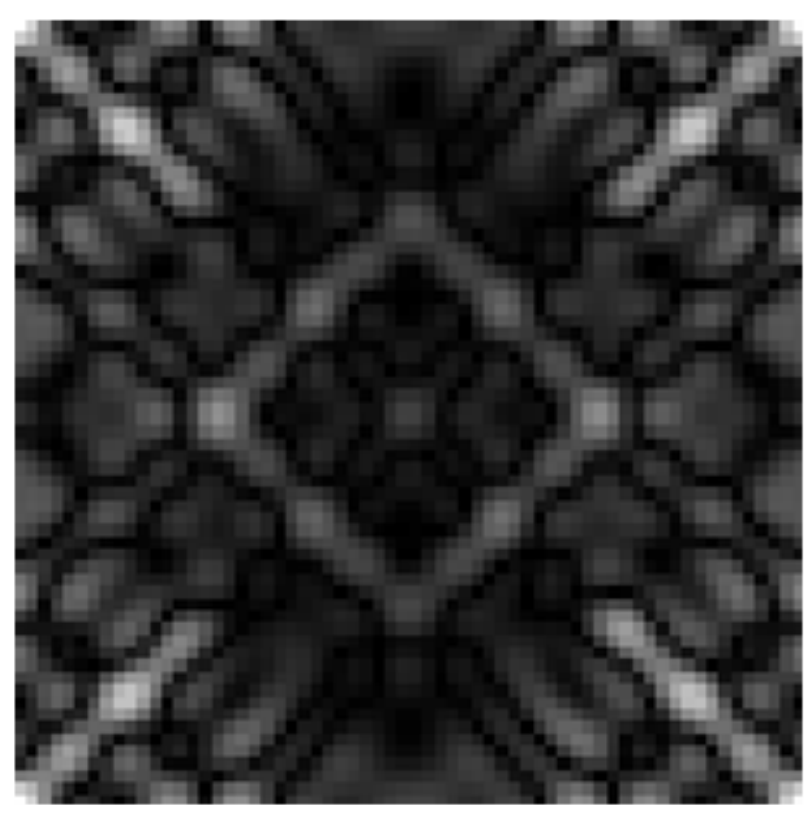

Figure 3. Raw image obtained by inverse Fourier transform of visibility function.

cross-like image which is seen as center of galaxies in real data.

\section{Conclusion}

In this paper we proposed an optimal hybrid method for deconvolution of VLBI images. In the proposed method, we take advantage from maximum entropy and compressed sensing methods and PSO algorithm 


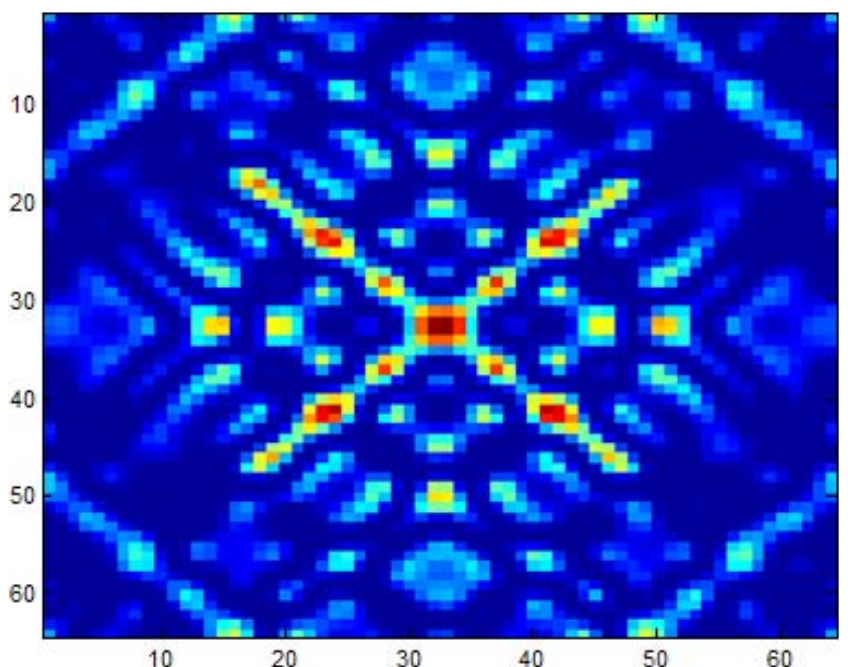

Figure 4. Image of $\mathrm{X}$ shape radio source after deconvolution by hybrid method.

for optimization. By performing the hybrid method for a simulated example in which the information is very limited in raw data we showed that the propose method is capable of recovering the main characteristics of source by using very simple strategies and no need to take special assumptions. One of the main advantages of using hybrid method which is also optimized by an algorithm like PSO is to get more confident of final results as different concepts are integrated. The proportions of weights in the defined fitness function could be used to get more understanding of data. For example: the results in the simulation section of this paper showed the higher weight for entropy term which could be interpreted as existence of a well defined probability density. Further study may be done for developing adaptive fitness functions dependent to data quality. This will make the system more flexible and reliable. For future research, the utilization of other optimization methods is also could be surveyed.

\section{References}

1. Bajkova A T, (2008). Multi-frequency synthesis of VLBI images using a generalized maximum entropy method, Astronomy Reports, vol 52(12), 951-962.

2. Banks A, Vincent $J$ et al. (2007). A review of particle swarm optimization. Part I: background and development, Natural Computing, vol 6(4), 467-484.

3. Banks A, Vincent $\mathrm{J}$ et al. (2008). A review of particle swarm optimization. Part II: hybridisation, combinatorial, multicriteria and constrained optimization, and indicative applications, Natural Computing, vol 7(1), 109-124.

4. Boroujeni S M S, Hemmati R et al. (2011). Optimal PID power system stabilizer tuning based on particle swarm optimization, Indian Journal of Science and Technology, vol 4(4), 379-383.

5. Clark B G (1980). An efficient implementation of the algorithm'CLEAN', Astronomy and Astrophysics, vol 89(3), 377-378.

6. Doeleman S S, Weintroub J et al., (2008). Event-horizonscale structure in the supermassive black hole candidate at the Galactic Centre, Nature, vol 455(7209), 78-80.

7. Donoho D L (2006). Compressed sensing, IEEE Transactions on Information Theory, vol 52(4), 1289-1306.

8. Dorigo M, Birattari M et al. (2006). Ant Colony Optimization, Computational Intelligence Magazine, IEEE, vol 1(4), 28-39.

9. Flacke, H, Melia F et al. (2008). Viewing the shadow of the black hole at the galactic center, The Astrophysical Journal Letters, vol 528(1), L13.

10. Goldberg D E, and Holland J H (1988). Genetic algorithms and machine learning, Machine Learning, vol 3(2-3), 95-99.

11. Jin Q, Wang G et al. (2010). Blind signal separation by entropy maximization(INFOMAX).20106th International Conference on Wireless Communications Networking and Mobile Computing (WiCOM), (pp. 1-5).

12. Kennedy J, and Eberhart R (1995). Particle swarm optimization, IEEE International Conference on Neural Networks , Proceedings., vol 4, 1942-1948.

13. Li F, Cornwell T J et al. (2011). The application of compressive sampling to radio astronomy, Astronomy and Astrophysics, vol 528.

14. Middelberg, E, and Bach U (2008). High resolution radio astronomy using very long baseline interferometry, Reports on Progress in Physics, vol 71(6), 066901.

15. Ramesh, N, and Nityananda R (1986). Maximum entropy image restoration in astronomy, Annual review of astronomy and astrophysics, vol 24, 127-170.

16. Readhead A C S, and Wilkinson P N (1978). The mapping of compact radio sources from VLBI data, The Astrophysical Journal, part 1, vol 223, 25-36.

17. Suksmono A B (2009). Deconvolution of VLBI images based on compressive sensing, ICEEI'09. International Conference on Electrical Engineering and Informatics, vol 1, 110-116.

18. Thompson A R, Moran J M et al. (2008). Interferometry and Synthesis in Radio Astronomy, John Wiley \& Sons.

19. Tsaig Y, and Donoho D L (2006). Extensions of compressed sensing, Signal processing, vol 86(3), 549-571. 